\title{
Ueber Blaps armeniaca Faldrm. und Bl. armeniaca All.
}

Von E. Ballion in Novorossisk (Russland).

$\mathrm{F}$ a ld e rma $\mathrm{n}$ beschreibt in seiner Fauna transcaucasica, II, pag. 42, Nr. 313, diese Art sehr ausführlich und kenntlich und bildet sie auf Tafel 7, Fig. 2 ziemlich gut ab. Demungeachtet wurde dieser Käfer von vielen Entomologen verkannt und mit anderen nahestehenden Arten verwechselt.

Fischer sagt in seinem Spicilegium, pag. 79, bei Blaps gages, dass die $B l$. armeniaca Faldrm. von dieser kaum oder gar nicht zu unterscheiden sei. Es ist aber noch sehr fraglich, ob Fischer auch die echte armeniaca vor sich gehabt habe; denn die $B l$. gages wurde nicht selten als armeniaca versandt. Ich habe auch eine gages als armeniaca aus der Krim zugeschickt bekommen. Im Bulletin des Naturalistes de Moscou, 1871, I, pag. 157, äusserte ich einige Zweifel über die Selbstständigkeit der $B l$. armeniaca und sprach die Vermuthung aus, dass armeniaca möglicherweise das Männchen von $B l$. taeniolata Mén. sein könnte. In Horae Soc. Entom. Rossicae, XI, im Artikel : „Beiträge zur Kenntniss der Käfer des europäischen und asiatischen Russlands" bezweifelt Fa ust auch die Artrechte der $B l$. armeniaca und betrachtet dieselbe als Varietät der $B l$. ominosa Mónét. Ich glaube aber, dass Herr $\mathrm{F}$ a ust sich irrt. Das Männchen von $B l$. armeniaca hat einen Haarbüschel am Bauche; das Männchen von $B l$. ominosa aber keinen, und schon deshalb können diese beiden Arten nicht vereinigt werden. Wenn ich mich früher irrte; so lag die Schuld am Mangel von Material und richtig bestimmter Käfer.

In den Annales d. J. Soc. entom. d. France, 1881, pag. 170, beschreibt Allard auch eine $B l$. armeniaca und citirt Fald., Fn. transc., H, 1837, pag. 42 (die Abbildung führt er nicht an) und setzt als Synonym hinzu auf der ersten Zeile isolirt: $B l$. plana Sol., Stud. ent., pag. 338, und auf der zweiten Zeile: $B l$. hians Fisch.; Spicil., pag. 78. - Muls., Latig., 1854, pag. 112.

Aus dieser Zusammenstellung der Citate muss man schliessen, dass $\mathrm{M}$ ulsant auch eine Bl.hians beschreibt; dem ist aber nicht so, denn $\mathrm{Mulsant}$ beschreibt hier die Soliersche Art.

Blaps plana ist mir nicht in natura bekannt. Ge $\mathrm{m} m$ in $\mathrm{g}$ e $\mathrm{r}$ und Harold führen sie in ihrem Kataloge (pag. 1864) als 
selbstständige Art an. Solier vergleicht seine $B l$. plana mit Bl. magica Er. und sagt, dass sie vielleicht nur locale Varietät der letzteren sei. $\mathrm{Mulsant}$ vergleicht sie auch mit $B l$. magica und gibt auch die Merkmale an, wodurch sie sich von $B l$. gigas und producta unterscheidet. Allard, welcher die plana Sol. armeniaca nennt, vergleicht sie mit Bl. taeniolata Ménét. und polychresta Forsk.

Diese Verschiedenheit der Ansichten bewog mich, die Beschreibungen bei Faldermann, Solier, Fischer, Mu]. sant und Allard genauer mit einander zu vergleichen.

Die Beschreibung der $B l$. armeniaca bei Faldermann stimmt nicht mit der Beschreibung der $B l$. plana bei Solier. Der Hauptunterschied liegt in der Form des Flügeldeckenfortsatzes. Nach Faldermann ist dieser lang, schmal, parallel und einzeln abgerundet. Solier beschreibt den Fortsatz bei plana als sehr kurz und in zwei scharfe Zähnchen getheilt beim Männchen, beim Weibchen beschränkt sich der Fortsatz nur auf zwei Zähnchen. Augenscheinlich, dass beide Autoren zwei verschiedene Arten beschreiben.

Die Beschreibung der $B l$. plana bei $\mathrm{Muls}$ ant ist bedeutend ausführlicher als bei Solier, stimmt aber im Wesentlichen mit jener überein. Von dem Flügeldeckenfortsatze des Männchens sagt auch Mulsant: très court, bidenté (ordinairement $11 /{ }_{10} \mathrm{~mm}$ ), beim Weibchen: prolongement caudal pres. que nul.

Die Grössenangabe ist bei allen drei Autoren fast gleich: lang $32-34 \mathrm{~mm}$, breit $13-14 \mathrm{~mm}$, Allard hingegen gibt die Grösse auf 29-37 mm Länge und $10^{1 / 2}-16 \mathrm{~mm}$ Breite an. Von dem Flügeldeckenfortsatz sagt Allard: ô prolongement caudal assez grêle et bifide $(3 \mathrm{~mm})$; $q$ Prolongement caudal plus court $\left(1_{1 / 2}^{1 /} \mathrm{mm}\right)$.

Was nun die Beschreibung der armeniaca betrifft, so hat Herr Allard es sich sehr leicht gemacht; denn er hat buchstäblich, fast mit photographischer Genauigkeit, Mulsant abgeschrieben. Die Beschreibung bei Faldermann hat er nicht der Beachtung gewürdigt. - Bei seiner Bl. armeniaca citirt Allard noch als Synonym: Bl. hians Fisch. Wie Allard darauf gekommen ist, begreife ich nicht.

$\mathrm{Ob} B l$. hians eine gute Art sei oder nicht, kann ich hier nicht untersuchen. Gemminger und $\mathrm{Harold}$ führen sie in 
ihrem Kataloge als selbstständige Art an. A llard hat dieses nicht beachtet und einen wahrscheinlich falsch bestimmten Käfer ohne Weiteres als Synonym zu seiner armeniaca sein sollenden Art gesetzt. Hätte Allard bei Fischer nachgelesen, so hätte er gefunden; dass die Beschreibung der $B l$. hians: "Thorax subquadratus, pulvinatus, punctulatus; elytra elongato-ovata, rugosa, sutura paulo elevata subhiante, mucronata, mucrone ramis fortibustriangularibus supra subhiantibus" sehr schlecht auf $B l$. plana Sol. oder auf $B l$. armeniaca Faldrm. passen.

Die Abbildung, welche Allard gibt, ist nicht nach der Natur gemacht und passt weder zu plana Sol,, noch zu $B l$. armeniaca Faldrm. Bei letzterer ist jede Hälfte des Fortsatzes einzeln abgerundet; in der Zeichnung bei Allard endet der Fortsatz mit zwei scharfen Spitzen.

Aus allem diesen erhellt, wie ich glaube, dass $B l$. armeniaca All. gar nicht die armeniaca Faldermann's ist, auch kann sie nicht als plana Sol. betrachtet werden; denn die von Allard der plana zugeschriebenen Fortsätze schaffen ein Mixtum compositum, ein Phantasiestück. Auch die Angabe der Fundorte scheint mir nicht ganz zuverlässig zu sein. Wenn er Bl. hians zu seiner Art stellt, warum gibt er nicht Podolien als Vaterland an?

\section{Note on the gen. Emypsara and Eudaemonius}

By George Lewis F. L. S. (Wimbledon).

1. A small series of Emypsara Adamsi Pascoe, that I brought from Japan, shows that it is a very variable insect as regards colour. Sometimes the elytra are wholly black and sometimes almost wholly pale. Emypsara flexuosa Pasc. is one of the varieties and Phaleria Hilgendorfi Harold is also the same species. I obtained about 14 specimens on the sands near Hakodate in 1880 .

2. I find that the generic name of Eudaemonius I used for an Erotylian in the Annals Mag. Nat. Hist. 1887, pag. 72, cannot stand as Eudaemonia has been used for a Lepidopterous insect. I wish therefore to substitute Eutriplax for Eudaemonius. 


\section{$2 \mathrm{BHL}$ Biodiversity Heritage Library}

Ballion, Ernst. 1887. "Ueber Blaps armeniaca FALDRM. und BI. armeniaca ALL." Wiener entomologische Zeitung 6, 307-309.

https://doi.org/10.5962/bhl.part.17770.

View This Item Online: $\underline{\text { https://www.biodiversitylibrary.org/item/44060 }}$

DOI: https://doi.org/10.5962/bhl.part.17770

Permalink: https://www.biodiversitylibrary.org/partpdf/17770

\section{Holding Institution}

Smithsonian Libraries

\section{Sponsored by}

Smithsonian

\section{Copyright \& Reuse}

Copyright Status: NOT_IN_COPYRIGHT

This document was created from content at the Biodiversity Heritage Library, the world's largest open access digital library for biodiversity literature and archives. Visit BHL at https://www.biodiversitylibrary.org. 